
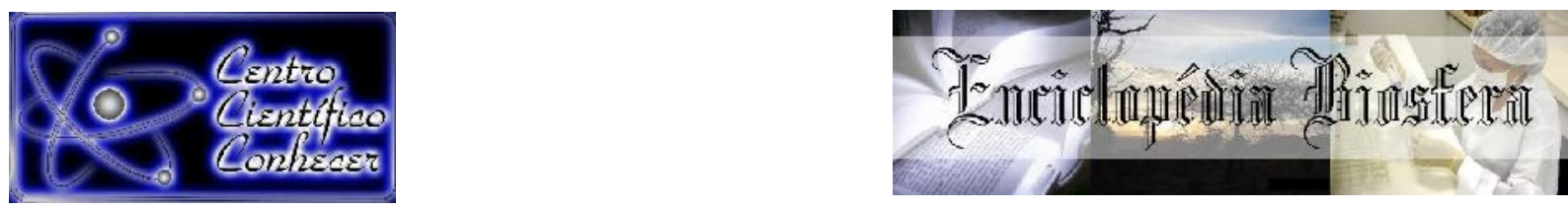

\title{
PERFIL SOCIODEMOGRÁFICO DE MULHERES COM DIAGNÓSTICO DE SÍFILIS CONGÊNITA ASSISTIDAS NA ESTRATÉGIA SAÚDE DA FAMÍLIA DE GOVERNADOR VALADARES/MG NO PERÍODO DE 2010 a 2018
}

\footnotetext{
Beatriz Alves De Pinho Bicalho ${ }^{\text {; }}$ Leonardo Oliveira Leão e Silva ${ }^{2}$; Valéria de Oliveira Ambrósio ${ }^{3}$; Marileny Boechat Frauches Brandão ${ }^{4}$.

${ }^{1}$ Acadêmica de enfermagem e bolsista de iniciação científica/ Universidade Vale do Rio Doce/UNIVALE; beatriz.faculdade@gmail.com.

${ }^{2}$ Prof. Adjunto do curso de Medicina e do Mestrado em Gestão Integrada do Território/Universidade Vale do Rio Doce. Doutorado em Saúde Coletiva pela Universidade Federal do Rio de Janeiro

${ }^{3}$ Prof. do curso de Enfermagem da Universidade Vale do Rio Doce. Mestrado em Gestão de Serviço de Saúde/Universidade Federal de Minas Gerais

${ }^{4}$ Prof. Adjunto do curso de Odontologia e do Mestrado em Gestão Integrada do Território/Universidade Vale do Rio Doce. Doutorado em Odontopediatria pela Universidade Cruzeiro do Sul.
}

\section{Recebido em: 15/02/2021 - Aprovado em: 15/03/2021 - Publicado em: 30/03/2021 DOI: 10.18677/EnciBio_2021A19}

\begin{abstract}
RESUMO
Introdução: A sífilis é um problema de saúde pública, que apresenta alta prevalência. A transmissão é por via sexual e vertical, raramente por transfusão sanguínea. A sífilis congênita (SC) é a infecção do feto pelo Treponema pallidum por via transplacentária, podendo ocorrer em qualquer fase da gestação, mas sendo evitada quando o diagnóstico ocorre precocemente. Objetivo: Caracterizar o perfil sociodemográfico e assistência pré-natal ofertada a mulheres com diagnóstico de sífilis congênita assistidas na Estratégia Saúde da Família de Governador Valadares/MG no período de 2010 a 2018. Metodologia: Estudo observacional, descritivo, com abordagem quantitativa. Os dados sobre os casos de SC foram obtidos de informações inseridas no banco de dados do Departamento de Informática do Sistema Único de Saúde e do Sistema de Informação de Agravos de Notificação. As análises das variáveis foram processadas com o software $\mathrm{R}^{\circledR}$ e expressas por meio de estatística descritiva, com os resultados apresentados por meio de frequência absoluta e relativa. Resultados: Observou-se que a maioria $(52,4 \%)$ das mulheres deste estudo apresentavam entre 20 a 29 anos, eram de cor parda (52,8\%) e apresentavam escolaridade ignorada $(69,0 \%)$. Apesar da maioria $(84,6 \%)$ das mulheres ter realizado o pré-natal, e o diagnóstico da SC ter sido realizado durante a gestação $(60,8 \%)$, o tratamento não ocorreu de forma adequada em 68,0\% dos casos. Conclusão: Para o combate à transmissão transplacentária da sífilis é fundamental a atuação da Atenção Básica durante o prénatal e a realização de novos estudos com foco em aprimorar a qualidade da assistência pré-natal.
\end{abstract}

PALAVRAS-CHAVE: assistência pré-natal, sífilis, sífilis congênita. 


\title{
SOCIODEMOGRAPHIC PROFILE OF WOMEN WITH CONGENITAL SYPHILIS DIAGNOSIS ASSISTED IN THE FAMILY HEALTH STRATEGY OF GOVERNADOR VALADARES / MG FROM 2010 TO 2018
}

\begin{abstract}
Introduction: Syphilis is a public health problem, which has a high prevalence. Transmission is by sexual and vertical route, rarely by blood transfusion. Congenital syphilis (CS) is infection of the fetus by Treponema pallidum transplacentally, which can occur at any stage of pregnancy, but is avoided when diagnosis occurs early. Objective: To characterize the sociodemographic profile and prenatal care offered to women diagnosed with congenital syphilis assisted in the Family Health Strategy of Governador Valadares/MG from 2010 to 2018. Methodology: Observational, descriptive study with quantitative approach. Data on cases of CS were obtained from information inserted in the database of the Informatics Department of the Unified Health System and the Notifiable Diseases Information System. The analyses of the variables were processed with the Software $R \circledast$ and expressed by descriptive statistics, with the results presented by means of absolute and relative frequency. Results: It was observed that the majority (52.4\%) women in this study were between 20 and 29 years old, were brown (52.8\%) and had ignored schooling (69.0\%). Despite the majority $(84.6 \%)$ women have had prenatal care, and the diagnosis of CS has been performed during pregnancy (60.8\%), treatment did not occur adequately in $68.0 \%$ of cases. Conclusion: For the fight against transplacental transmission of syphilis, it is essential to act primary care during prenatal care and to conduct further studies with a focus on improving the quality of prenatal care.
\end{abstract}

KEYWORDS: congenital syphilis, syphilis, prenatal care.

\section{INTRODUÇÃO}

A sífilis é uma patologia infectocontagiosa sistêmica, de evolução crônica, causada pela bactéria Treponema pallidum, sendo descoberta no século XV. Pode ser transmitida por via sexual, vertical e sanguínea. Embora de diagnóstico rápido e tratamento de baixo custo, ainda é considerada um problema de saúde pública devido aos elevados índices de casos (SILVA et al., 2017; REIS et al., 2018).

Quando a sífilis não é tratada ou o esquema de tratamento ocorre de forma inadequada na gestante, essa infecção pode ser transmitida para o concepto por via transplacentária, ocasionando a sífilis congênita (SC). Essa doença pode ser classificada em precoce ou tardia, levando-se em consideração o surgimento da síndrome clínica, sendo respectivamente, antes e depois dos dois anos de vida (LIMA et al., 2017).

A SC é uma doença de notificação compulsória desde 22 de dezembro de 1986 por meio da Portaria Ministerial $N^{\circ} 542$. Já a sífilis na gestante passou a constar entre as doenças e agravos de notificação compulsória a partir de 2005, por meio da Portaria Ministerial $N^{\circ} 33$ de 14 de julho desse mesmo ano (LIMA et al., 2017).

Para diminuição dos casos de SC, a Organização Pan-Americana da Saúde (OPAS) propôs renovar e articular as estratégias de enfrentamento, definindo como meta para o ano de 2020 uma incidência de 0,5 casos/mil nascidos vivos. Com finalidade de alcançar essa meta, são necessárias novas estratégias que ampliem a 
cobertura da testagem de gestantes e mulheres em idade fértil e o tratamento imediato dos casos positivos (HERINGER et al., 2020).

A adequada assistência pré-natal durante a gravidez é a medida de controle mais eficiente, sendo necessária a captação precoce da gestante e a realização de, no mínimo, seis consultas, com triagem sorológica (VDRL), no primeiro trimestre da gestação, e repetição (segundo teste), em torno da 28ª semana. Na condição de testes reagentes positivos, deve ser feita a busca ativa das gestantes com o intuito de tratamento e acompanhamento (VIANNA et al., 2017).

As condições associadas à ocorrência de sífilis na gestação são inúmeras, dentre estas, fatores sociodemográficos, comportamentais e os relacionados à assistência à saúde. Dentre os fatores sociodemográficos, a pouca escolaridade, baixa renda e situação conjugal são apontadas como situações de risco e uma expressão de que a sífilis se relaciona com a pobreza, embora não se limite a esta condição. Alguns dos comportamentos que vulnerabilizam as mulheres, associando-se o maior risco, como início precoce de relação sexual e da gestação, a multiplicidade de parceiros sexuais, recusa no uso de preservativos, uso de drogas ilícitas e psicoativas, entre outros. Algumas dessas condições ampliam o risco ao se relacionar com o insuficiente acesso aos serviços de saúde (MELO et al., 2011; DOMINGUES et al., 2013).

Mediante a relevância da doença como um problema de saúde pública e a elevada incidência no Brasil e no mundo, o presente estudo teve como objetivo caracterizar o perfil sociodemográfico e a assistência pré-natal ofertada a mulheres com diagnóstico de sífilis congênita assistidas na Estratégia Saúde da Família (ESF) de Governador Valadares/MG no período de 2010 a 2018.

\section{MATERIAL E MÉTODOS}

Tratou-se de um estudo observacional, descritivo, com abordagem quantitativa sobre a incidência de casos de sífilis congênita no período de 2010 a 2018 em Governador Valadares/MG. O município de Governador Valadares possuía uma população estimada de 278.685 habitantes, em 2018, distribuídos em $2.342,325 \mathrm{Km}^{2}$, com Índice de Desenvolvimento Humano (IDH) de 0,727. É considerado um município de médio porte, em desenvolvimento.

Encontra-se localizado no leste de Minas Gerais; é pertencente à Bacia do Rio Doce, com 183 bairros na zona urbana e 12 distritos na zona rural (IBGE, 2018). Os dados em relação à SC foram obtidos a partir das informações inseridas no banco de dados do Departamento de Informática do Sistema Único de Saúde (DATASUS Ministério da Saúde) e Sistema de Informação de Agravos de Notificação (SINAN). O DATASUS contém um sistema de informações em saúde on-line que pode ser consultado no website http://www.datasus.gov.br.

O DATASUS disponibiliza informações que contribuem para subsidiar análises objetivas da situação sanitária, tomada de decisão baseadas em evidências e elaboração de programas de ações de saúde. Por meio dessa ferramenta é possível obter os dados de forma rápida, para acesso às informações de saúde diversas da população (BRASIL, 2020). As seguintes variáveis foram utilizadas: faixa etária, raça/cor, escolaridade, realização do pré-natal, momento de diagnóstico da sífilis materna, esquema de tratamento materno, parceiro tratado. Os dados foram coletados e analisados no período de 25 de junho de 2020 a 31 de dezembro de 2020. As 
análises destas variáveis foram processadas com o uso do software $R^{\circledR}$ (versão 3.2.2) e expressas por meio de estatística descritiva. Os resultados foram apresentados por meio de frequência absoluta e relativa das respostas.

\section{RESULTADOS E DISCUSSÕES}

No período de 2010 a 2018 foram notificados e diagnosticados 403 casos de SC no município de Governador Valadares/MG, com uma taxa de detecção de 11,4/mil nascidos vivos (Gráfico 1). Sendo que destes, 375 (93,0\%) foram diagnosticados com SC recente e nenhum caso tardio, no entanto ocorreram seis $(1,49 \%)$ abortos e 22 $(5,46 \%)$ natimortos. Observou-se que em Governador Valadares/MG ocorreu um aumento no número de casos de SC em 2012, entretanto verificou-se uma queda em 2016, mas em contrapartida a partir de 2017 os números de casos voltaram a subir significativamente.

GRÁFICO 1 - Número de casos notificados e a taxa de detecção/mil nascidos vivos de SC em Governador Valadares/MG, 2010 - 2018.

W $N^{i}$ de casos Laxas de detecçào

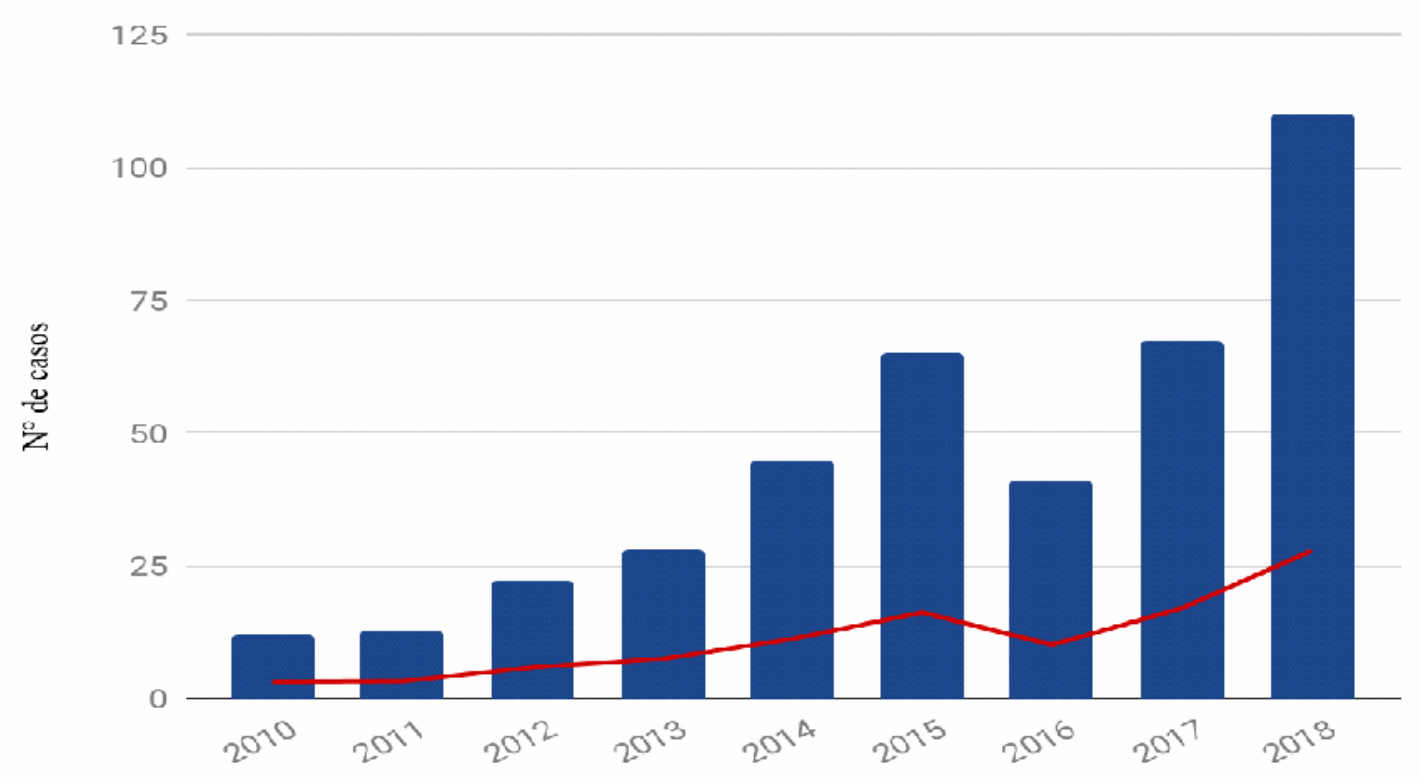

Fonte: Ministério da Saúde/SVS - Sistema de Informação de Agravos de Notificação - SINAN Net (2020)

Vale salientar que no estado de Minas Gerais no período de 2008 a 2018, observou-se também um aumento substancial da SC, a partir do ano de 2011, passando de 312 casos para mais de 1877 casos no ano de 2017 (Gráfico 2). 
GRÁFICO 2 - Número absoluto, Incidência e Letalidade da Sífilis Congênita no estado de Minas Gerais, 2008 a 2018.

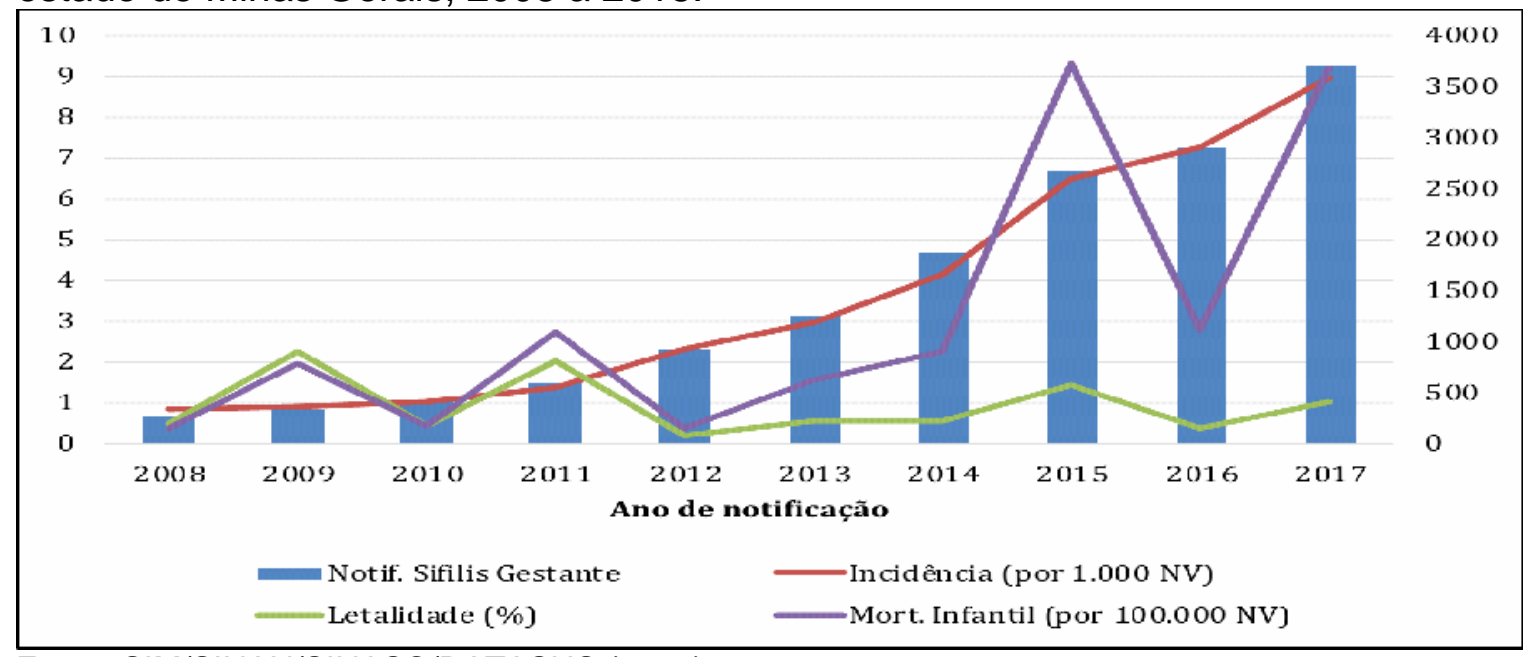

Fonte: SIM/SINAN/SINASC/DATASUS (2020)

Saraceni et al. (2017) evidenciaram também um elevado número de casos de SC, notificados entre 2007 e 2012, nos estados de Rio de Janeiro, Ceará e Rio Grande do Sul com, respectivamente, 9.820, 4.045 e 2.745. Entretanto Soeiro et al. (2014) registraram um menor número de casos, pesquisando a SC no estado de Amazonas, verificou-se no ano de 2008 a ocorrência de 193 casos e em 2009, 140 casos.

Heringer et al. (2020) pesquisando a SC no município de Niterói/RJ, no período de 2007 a 2016 observaram 754 casos notificados de SC obtendo um resultado semelhante na classificação final, com predomínio de SC recente $(86,9 \%)$, seguida de abortos $(6,8 \%)$ e natimortos (5\%).

O Ministério da Saúde (MS) juntamente com a OPAS definiram como meta a redução da SC para menos de 0,5 casos/mil nascidos vivos até o ano de 2015, para que a doença seja considerada eliminada como um problema de saúde pública (LIMA, et al., 2017). Mas verificou-se que com o passar do tempo, os casos aumentaram, possivelmente devido à uma assistência pré-natal inadequada.

A incidência de SC em Minas Gerais vem aumentando, sendo que em 2008 apresentava menos de 1,0/mil nascidos vivos e em 2017 atingiu o registro de mais de 9,0/mil nascidos vivos. Ferreira et al. (2018) pesquisando a incidência de SC no período de 2010 até 2015, a partir dos informes epidemiológicos do estado do Rio de Janeiro observaram um valor de 7 para 13,6 casos/mil nascidos vivos. Todavia Amâncio et al. (2016) pesquisando a SC no estado de Goiás no período de 2008 a 2013 verificaram 538 casos, sendo que a incidência variou entre 6 (2013) a 1,2 (2012) por mil nascidos vivos, considerando uma menor incidência entre os estudos acima apresentados, mas ainda não está dentro da meta exigida pelo MS e a OPAS.

Ressalta-se ainda que no período citado (Gráfico 2), a letalidade em Minas Gerais apresentou oscilações, sendo que em 2008 era menos de $1 \%$ e já em 2009 aumentou para mais de $2 \%$ e nos anos seguintes se manteve entre essas porcentagens. A mortalidade infantil por SC nos anos de 2008 a 2010 em Minas Gerais teve variações, considerando que em 2008 era 2,0/cem mil nascidos vivos e chegando 
a mais de 9,0/cem mil nascidos vivos em 2015 e ocorreu uma queda novamente em 2016. Taxas semelhantes foram encontradas por Canto et al. (2019), pesquisando a SC no Ceará, quando observaram no período de 2010 a 2014 o total de 24 óbitos infantis notificados relatados ao SIM, com uma taxa de 3,8 mortes/cem mil nascidos vivos. Logo, em Governador Valadares a mortalidade infantil por SC teve uma taxa de 9,11/cem mil nascidos vivos entre 2008 e 2010.

No que se refere às características sociodemográficas maternas, a tabela 1 demonstra o perfil epidemiológico das mulheres pesquisadas no período de 2010 a 2018 em Governador Valadares/MG. Observou-se que houve predominância de mulheres com diagnóstico de SC na faixa etária entre 20 a 29 anos $(52,4 \%)$, seguida da faixa etária de 15 a 19 anos (27,5\%), um baixo percentual nas faixas etárias abaixo de 14 anos e acima de 40 anos.

TABELA 1 - Distribuição das características maternas dos casos diagnosticados de SC em Governador Valadares/MG, 2010 - 2018.

\begin{tabular}{|c|c|c|}
\hline Características Maternas & $\mathrm{N}^{\circ}$ & $\%$ \\
\hline \multicolumn{3}{|l|}{ Faixa etária } \\
\hline 10 a 14 anos & 5 & 1,2 \\
\hline 15 a 19 anos & 111 & 27,5 \\
\hline 20 a 29 anos & 211 & 52,4 \\
\hline 30 a 39 anos & 59 & 14,6 \\
\hline 40 ou mais & 7 & 1,7 \\
\hline Ignorado & 10 & 2,5 \\
\hline \multicolumn{3}{|l|}{ Raça/Cor } \\
\hline Branca & 32 & 7,9 \\
\hline Preta & 35 & 8,7 \\
\hline Amarela & 1 & 0,2 \\
\hline Parda & 235 & 58,3 \\
\hline Ignorada & 100 & 24,8 \\
\hline \multicolumn{3}{|l|}{ Escolaridade } \\
\hline $1^{\text {a }}$ e $4^{\underline{a}}$ série incompleta & 5 & 1,2 \\
\hline $4^{\text {a }}$ série completa & 2 & 0,5 \\
\hline $5^{\mathrm{a}}$ a $8^{\mathrm{a}}$ série incompleta & 36 & 8,9 \\
\hline Fundamental completo & 22 & 5,5 \\
\hline Médio incompleto & 25 & 6,2 \\
\hline Médio completo & 27 & 6,7 \\
\hline Superior incompleto & 5 & 1,2 \\
\hline Superior completo & 2 & 0,5 \\
\hline Não se aplica & 1 & 0,2 \\
\hline Ignorado & 278 & 69 \\
\hline
\end{tabular}

Fonte: Ministério da Saúde/SVS - Sistema de Informação de Agravos de Notificação - SINAN Net (2020)

Silva et al. (2017) encontraram maior prevalência da infecção de sífilis associada à faixa etária de 16 a 30 anos. Já Lima et al. (2017) nos anos de 2008 a 2013, no município de Sobral/CE verificaram 119 casos notificados e diagnosticados de SC com predominância na faixa etária de 20 a 25 anos. Machado et al. (2018) pesquisando os ENCICLOPÉDIA BIOSFERA, Centro Científico Conhecer - Jandaia-GO, v.18 n.35; p. 255 
óbitos perinatais por SC no Brasil no período de 2001/2002 e 2012/2013 observaram um acometimento maior na faixa etária média entre 20 a 34 anos $(61,4 \%)$ anos.

Acredita-se que o intervalo etário de maior dominância pode estar associado ao período considerado reprodutivo da mulher, assim, as mulheres jovens estão mais propensas a adquirir a infecção, por estarem sexualmente ativas. O comportamento sexual inadequado também pode ser considerado como fator de risco para contrair Infecções Sexualmente Transmissíveis (IST) devido a falta do uso de preservativos e multiplicidade de parceiros (SILVA et al., 2017).

Quanto a variável raça/cor, a maioria das mulheres pesquisadas se autoreferiram de cor parda (58,3\%). Rodrigues et al. (2018) no período de 2007 a 2013 coletaram dados de casos notificados no município de Sobral/CE, sendo no total de 120 casos de SC em uma ESF, obtiveram um resultado semelhante de $111(92,5 \%)$ de mães pardas. Tal resultado corrobora os resultados de Silva et al. (2017); Lima et al. (2017) e Heringer et al. (2020) cuja maior predominância da SC foi entre mães pardas. Entretanto, Silva Neto et al. (2018) em um estudo com 149 gestantes em Itapeva/SP no período de 2010 a 2014 obtiveram um resultado diferente, havendo um acometimento maior em mães brancas.

Considerando a escolaridade, observou-se que $36(8,9 \%)$ das mulheres deste levantamento estudaram até a $5^{\underline{a}}$ a $8^{\underline{a}}$ série incompleta do Ensino Fundamental. Vale ressaltar que o número de casos de mulheres com registro de escolaridade ignorada mostrou-se elevado, 278 (69,0\%). Lima et al. (2017) e Rodrigues et al. (2018) obtiveram números mais altos em relação ao do estudado, sendo respectivamente, $43,0 \%$ e $35,8 \%$ das mulheres com ensino fundamental incompleto. Entretanto Domingues et al. (2013) pesquisando sobre SC em 2.422 gestantes durante o acompanhamento pré-natal em unidades de saúde da rede SUS do município do Rio de Janeiro nos anos 2007 e 2008, obtiveram 410 (36,5\%) com ensino médio. Resultados reportados por Reis et al. (2018) pesquisando a situação da SC e seus determinantes, expresso no período de 2011-2014 na cidade do Rio de Janeiro, observaram que $41,0 \%$ apresentavam dados de escolaridade ignorada.

No que tange às características da assistência pré-natal verificou-se que 341 $(84,6 \%)$ das gestantes realizaram pré-natal, no entanto, $37(9,2 \%)$ não, e $25(6,2 \%)$ ignorados (tabela 2). O referido achado vai ao encontro dos resultados de Cavalcante et al. (2017), que identificaram 171 casos de sífilis em gestantes e 204 casos de SC no período de 2007 a 2014 em Palmas, quando 81,34\% realizaram o pré-natal, 17,2\% não realizaram e em $1,5 \%$ os dados foram ignorados/em branco.

TABELA 2 - Distribuição das características da assistência pré-natal das mães dos recém-nascidos diagnosticados com SC em Governador Valadares/MG, 2010 - 2018.

\begin{tabular}{|l|c|c|}
\hline Características da assistência pré-natal & $\mathrm{N}$ & $\%$ \\
\hline Realização de pré-natal & & \\
\hline Sim & 341 & 84,6 \\
Não & 37 & 9,2 \\
Ignorado & 25 & 6,2 \\
\hline Momento do diagnóstico da sífilis materna & & \\
\hline Durante o pré-natal & 245 & 60,8 \\
No momento do parto/curetagem & 80 & 19,9 \\
\hline
\end{tabular}




\begin{tabular}{|l|c|c|}
\hline Após o parto & 38 & 9,4 \\
Não realizado & 3 & 0,7 \\
Ignorado & 37 & 9,2 \\
\hline Esquema de tratamento materno & & \\
\hline Adequado & 9 & 2,2 \\
Inadequado & 274 & 68,0 \\
Não realizado & 68 & 16,9 \\
Ignorado & 52 & 12,9 \\
\hline Parceiro tratado & & \\
\hline Sim & 33 & 8,2 \\
Não & 197 & 48,9 \\
Ignorado & 173 & 42,9 \\
\hline
\end{tabular}

Fonte: Ministério da Saúde/SVS - Sistema de Informação de Agravos de Notificação - Sinan Net (2020)

A acessibilidade ao pré-natal no primeiro trimestre é um indicador de qualidade da Atenção Básica, portanto, na busca por melhora na qualidade da assistência, o governo federal implantou o Programa Rede Cegonha, com o objetivo de oferecer acolhimento de qualidade a todas as gestantes atendidas pelo Sistema Único de Saúde (SUS), desde a descoberta da gestação até 24 meses da criança. Destaca-se que com o Programa vieram os testes rápidos para HIV, Sífilis, Hepatite B e C, de forma a proporcionar o diagnóstico precoce e tratamento em tempo oportuno, para prevenir a transmissão vertical para o feto (SILVA, et al., 2017; RODRIGUES, et al., 2018).

O momento de diagnóstico da sífilis materna ocorreu para a maioria das mulheres deste estudo durante a gestação- 245 (60,8\%), porém 80 (19,9\%) descobriram no momento de parto/curetagem, $38(9,4 \%)$ após o parto, $3(0,7 \%)$ não tiveram diagnóstico e $37(9,2 \%)$ ignorados. Estes achados assemelham-se aos resultados reportados por Reis et al. (2018), Rodrigues et al. (2018); Souza et al. (2018) e Heringer et al. (2020).

A assistência pré-natal é fundamental para diagnosticar alterações na gestação de forma precoce, sendo possível realizar intervenções efetivas para reduzir os riscos à saúde da gestante e do feto (SILVA et al., 2017). No entanto, observou-se que mesmo com a realização do pré-natal e diagnóstico durante a gestação ainda ocorreu transmissão vertical da sífilis. Assim, segundo Amâncio et al. (2016), Rodrigues et al. (2018) e Heringer et al. (2020) as falhas no atendimento prestado durante o pré-natal ainda são muito visíveis e para resolvê-las é preciso principalmente, realizar medidas socioeducativas e melhorias nos serviços de saúde prestados na atenção básica.

Em vista disso, foram verificados avanços no diagnóstico durante o pré-natal, contudo, sugere-se a ocorrência de reinfecção das gestantes pelos parceiros não tratados ou tratamento inadequado da gestante, como também a reinfecção desses parceiros tratados com o contato sexual com outras mulheres infectadas e não tratadas (MAGALHÃES et al., 2013).

Entre as gestantes com o diagnóstico durante o pré-natal, 274 (68,0\%) foram tratadas inadequadamente, $68(16,9 \%)$ não realizaram o tratamento, 52 (12,9\%) ignorado e apenas 9 (2,2\%) tiveram tratamento adequado. Realidade semelhante também foi encontrada em outros estudos, alertando para falhas nas adequabilidades 
do tratamento dessas mulheres (SILVA et al., 2017; REIS et al., 2018; RODRIGUES et al., 2018).

Quanto ao tratamento, com o propósito de que a gestante portadora de sífilis seja considerada adequadamente tratada, afastando a possibilidade de infecção ao concepto, esta deve ser medicada com penicilina $G$ benzatina, nas doses apropriadas à fase da infecção, ter finalizado o tratamento pelo menos 30 dias antes do parto, e ter o parceiro concomitantemente tratado com o mesmo esquema terapêutico da grávida (CAMPOS et al., 2010).

Acredita-se que a sífilis é uma patologia que acomete uma população desfavorecida socialmente em que os fatores sociais são mais acentuados. Esse fator pode corroborar uma deficiência em seu autocuidado e comprometimento na realização do tratamento (LIMA et al., 2017; SOUZA et al., 2018).

Em relação ao tratamento do parceiro sexual, notou-se que apenas $33(8,2 \%)$ realizaram o tratamento, 197 (48,9\%) não o realizaram concomitantemente à gestante e $173(42,9 \%)$ eram dados ignorados. Situação semelhante foi encontrada por Reis et al. (2018) no Rio de Janeiro/RJ, onde $61,0 \%$ dos parceiros não foram tratados, $28 \%$ ignorados e somente $11 \%$ completou o tratamento.

Segundo Heringer et al. (2020) existem diferentes motivos para os parceiros não se tratarem, quer seja por sua própria recusa, desconfiança do diagnóstico, por estarem assintomáticos ou terem medo. Algumas vezes percebe-se que a própria gestante oculta o diagnóstico por temer afastamento definitivo do parceiro, ou para evitar conflitos no relacionamento, ou ainda por desconhecimento da importância do tratamento. Evidenciou-se, segundo Amâncio et al. (2016) que existe um desafio na assistência à saúde do homem, tendo uma necessidade de maior destaque por ser foco de políticas de saúde.

\section{CONCLUSÕES}

A partir destas análises, pôde-se concluir a necessidade de um aperfeiçoamento e capacitação dos profissionais de saúde na realização do pré-natal. Mesmo com um percentual grande de gestantes realizando o pré-natal, a assistência prestada foi realizada de maneira inapropriada e com baixa resolutividade. Desta forma, sugere-se com base nos resultados, a oferta de qualificação profissional para diagnóstico oportuno da sífilis em gestante, bem como 0 preenchimento adequado dos instrumentos de notificação.

Adicionalmente, oferta do tratamento adequado e educação em saúde das gestantes e seus parceiros. Torna-se indispensável os profissionais, após capacitação, exercerem seu papel de forma consciente, de maneira a minimizar os riscos desta doença totalmente evitável. Desse modo, espera-se que o combate à transmissão transplacentária da sífilis seja fortalecida com a atuação de qualidade da Atenção Básica à Saúde.

Por fim, compreende-se que ainda há um longo caminho a ser trilhado com o intuito de que os casos de sífilis e sífilis congênita diminuam e recomenda-se a realização de novos estudos com foco em aprimorar a qualidade da assistência prénatal. 


\section{REFERÊNCIAS}

AMÂNCIO, V. C.; GRACIANO, A. R.; COZER, A. M; ASSIS, L. P. F.; DIAS, D. C. S. Epidemiologia da sífilis congênita no Estado de Goiás. Revista Educação em Saúde. Goiás, v. 2, n. 4, p. 58-63, 2016. Disponível em: <https://core.ac.uk/download/pdf/234552279.pdf>. Acesso em:13 de fev. 2021.

BRASIL. Ministério da Saúde. Banco de dados do Sistema Único de Saúde. DATASUS. Disponível em: <http://tabnet.datasus.gov.br/cgi/tabcgi.exe?ibge/cnv/poptmg.def>. Acesso em: 11 de Fev. 2020.

CAMPOS, A. L. A.; ARAÚJO, M. A. L.; MELO, S. P; GONÇALVES, M. L. C. Epidemiologia da sífilis gestacional em Fortaleza, Ceará, Brasil: agravos sem controle. Cadernos de Saúde Pública; v. 26, n. 9, p. 1747-55, 2010. Disponível em: $<$ https://www.scielo.br/scielo.php?script=sci_arttext\&pid=S0102-311X2010000900008>. doi:10.1590/S0102-311X2010000900008. Acesso em: 13 de fev. 2021.

CANTO, S. V. E.; ARAÚJO, M. A. L.; MIRANDA, A. E.; CARDOSO, A. R. P.; ALMEIDA, R. L. F. Fetal and infant mortality of congenital syphilis reported to the Health Information System. PLOS ONE, v. 1, n. 14, p. 1-11, Jan/2019. Disponível em: <https://journals.plos.org/plosone/article?id=10.1371/journal.pone.0209906>. doi: 10.1371/journal.pone.0213214. Acesso em: 13 fev. 2021.

CAVAlCANTE, P. A. M. PEREIRA, R. B. L. CASTRO, J. G. D. Sífilis gestacional e congênita em Palmas, Tocantins, 2007-2014. Epidemiologia e Serviços de Saúde, Brasília, v. 26, n. 2, p. 255-264, abr/jun,2017. Disponível em: $<$ https://www.scielo.br/scielo.php?pid=S2237-

96222017000200255\&script=sci_abstract\&tlng=pt>.

49742017000200003. Acesso em: 13 fev. 2021.

doi:10.5123/s1679-

DOMINGUES, R. M. S. M.; SARACEN, V.; HARTZ, Z. M. A.; LEAL, M. C. Sífilis congênita: evento sentinela da qualidade da assistência pré-natal. Revista de Saúde Pública; v. 47, n. 1, p. 147-57, 2013. Disponível em: $<$ https://www.scielo.br/scielo.php?script=sci_arttext\&pid=S0034-89102013000100019>. doi:10.1590/S0034-89102013000100019. Acesso em: 13 fev. 2021.

FERREIRA, A. G, BARBOSA, V. F. J, MALECK, M. Comparação entre a incidência, nacional, estadual e do município de Vassouras, de sífilis congênita, nos últimos quinze anos. Revista de Saúde; v. 9, n. 1, p. 14-17, jan./jun. 2018. Disponível em: < http://editora.universidadedevassouras.edu.br/index.php/RS/article/view/1248>. doi:10.21727/rs.v9i1.1248. Acesso em: 13 fev. 2021.

HERINGER, A. L. S; KAWA, H.; FONSECA, S. C.; BRIGNOL, S. M. S.; ZARPELLON, L. A.; REIS, A. C. et al. Desigualdades na tendência da sífilis congênita no município de Niterói, Brasil, 2007 a 2016. Revista Panamericana Salud Pública, v. 8, n. 44, p. 1-8, 2020. em: 
https://www.researchgate.net/publication/339029977_Desigualdades_na_tendencia_da sifilis_congenita_no_municipio_de_Niteroi_Brasil_2007_a_2016>. 10.26633/RPSP.2020.8. Acesso em: 13 fev. 2021.

IBGE - Instituto Brasileiro de Geografia e Estatística. PAS - Pesquisa Anual de Serviços, 2018. [online]. Disponível em: https://censo.demográfico. ibge.gov.br/brasil/mg/governador-valadares/panorama. Acesso em: 21 de jan.de 2020.

LIMA, V. C.; MORORÓ, R. M.; MARTINS, M. A.; RIBEIRO, S. M.; LINHARES, M. S. C. Perfil epidemiológico dos casos de sífilis congênita em um município de médio porte no nordeste brasileiro. Journal of Health \& Biological Sciences. Sobral-CE, v. 1, n. 5, p. 56-61, 2017. Disponível em: < https://periodicos.unichristus.edu.br/jhbs/article/view/1012/406>. doi:10.12662/23173076jhbs.v5i1.1012. Acesso em: 13 fev. 2021.

MACHADO, C.J.; SIMÃO, A.B.; GONÇALVES, R.V.; AZEVEDO, A.C.; DRUMOND, E.F. et al. Sífilis congênita no Brasil em 2001/2002 e 2012/2013: estudo de causas múltiplas de óbito. Revista da Faculdade de Ciências Médicas de Sorocaba. Sorocaba; v. 20, n. $2, \quad$ p. $98-103,2018.2$ Disponível em: <https://revistas.pucsp.br/index.php/RFCMS/article/view/34914/pdf>. doi: 10.23925/1984-4840.2018v20i2a8. Acesso em: 13 fev. 2021.

MAGALHÃES, D. M. S.; KAWAGUCHI, I. A. L.; DIAS, A.; CALDERON, I. M. P. Sífilis materna e congênita: ainda um desafio. Cadernos de Saúde pública; v. 29, n. 6, p. 1109-20, 2013. Disponível em: <https://www.scielo.br/pdf/csp/v29n6/a08v29n6.pdf>. Acesso em: 13 fev. 2021.

MELO, N. G. D. O.; MELO, F. D. A.; FERREIRA, L. O. C. Diferenciais intraurbanos de sífilis congênita no Recife, Pernambuco, Brasil (2004-2006). Epidemiologia e Serviços de Saúde; v. 20, n. 2, p. 213-22, 2011. Disponível em: < http://scielo.iec.gov.br/pdf/ess/v20n2/v20n2a10.pdf>. doi: 10.5123/S167949742011000200010. Acesso em: 13 fev. 2021.

SILVA NETO, S. E.; SILVA, S. S. B. E.; SARTORI, A. M. C. Syphilis in pregnancy, congenital syphilis, and factors associated with mother-to-child transmission in Itapeva/SP, 2010 to 2014. Revista da Sociedade Brasileira de Medicina Tropical. Uberaba, v. 51, n. 6, p. 819- 826, Nov./Dec. 2018. Disponível em: < https://www.scielo.br/scielo.php?script=sci_arttext\&pid=S0037-86822018000600819>. doi: 10.1590/0037-8682-0377-2017. Acesso em: 13 fev. 2021.

REIS, G. J.; BARCELLOS, C.; PEDROSO, M. M; XAVIER, D. R. Diferenciais intraurbanos da sífilis congênita: análise preditiva por bairros do Município do Rio de Janeiro, Brasil. Cadernos de Saúde Pública. Rio de Janeiro, v. 9, n. 34, p. 1-13, 2018. Disponível em: < https://www.scielo.br/scielo.php?script=sci_arttext\&pid=S0102311X2018000905010.>. doi: 10.1590/0102-311x00105517. Acesso em: 13 fev. 2021. 
RODRIGUES, I. M.; RIBEIRO, M. A.; ALBUQUERQUE, I. M. N.; DIAS, L. K. S.; AGUIAR, N. L. T. et al. Perfil e distribuição espacial da sífilis congênita em Sobral-CE no período de 2007 a 2013. Ciência \& Saúde. Sobral-CE, v. 2, n. 11, p. 70-76, abr./jun. 2018.

SARACENI, V.; PEREIRA, G. F. M.; SILVEIRA, M. F.; ARAUJO, M. A. L.; MIRAND, A. E. Vigilância epidemiológica da transmissão vertical da sífilis: dados de seis unidades federativas no Brasil. Revista Panamericana de Salud Pública, v. 41, n. 44, p. 1-8, 2017. Disponível em: <https://www.scielosp.org/pdf/rpsp/2017.v41/e44>. Acesso em: 13 fev. 2021.

SILVA, L. C. V. G.; TEODORO, C. J.; SILVA, J. K.; SANTOS, D. A. S.; OLINDA, R. A. Perfil dos casos de sífilis congênita em um município do sul de Mato Grosso. Journal Health NPEPS (Núcleo de Pesquisa e Extensão Política, Planejamento, Organização e Práticas (individual e coletiva) em Saúde); v. 2, n. 2, p. 380-390, 2017. Disponível

em: <https://periodicos.unemat.br/index.php/jhnpeps/article/view/2656/2148>. Acesso em: 13 fev. 2021.

SOEIRO, C. M. O.; MIRANDA, A. E.; SARACENI, V.; SANTOS, M. C.; TALHARI, S. et al. Syphilis in pregnancy and congenital syphilis in Amazonas State, Brazil: an evaluation using database linkage. Cadernos de Saúde Pública. Rio de Janeiro, v.30, n.4, p.715-723, abr/2014. Disponível em: <https://www.scielo.br/scielo.php?script=sci_arttext\&pid=S0102-311X2014000400715>. doi: 10.1590/0102-311X00156312. Acesso em: 13 fev. 2021.

SOUZA, J. G. N.; OLIVEIRA, L. L.; LIMA, L. R.; BRASILINO, C. E. M.; HOLANDA, S. K. S. et al. Análise epidemiológica da sífilis congênita no sertão central Cearense de 2006 a 2015. Revista Expressão Católica Saúde, v. 3, n. 2, p. 18-23; jul.-dez, 2018. Disponível em: <http://publicacoesacademicas.unicatolicaquixada.edu.br/index.php/recsaude/article/vie w/2428>. doi: 10.25191/recs.v3i2.2428. Acesso em: 13 fev. 2021.

VIANNA, P. V. C.; HELBUStO, N. B.; BARBOSA, R. J.; SANTOS, M. H. S. Sífilis congênita, um evento sentinela: narrativas de mães de filhos nascidos com sífilis em uma cidade metropolitana paulista. Revista Univap. São José dos Campos-SP-Brasil, v. 23, n. 42, jul/2017. Disponível em: <https://revista.univap.br/index.php/revistaunivap/article/view/1779>. 10.18066/revistaunivap.v23i42.1779. Acesso em: 13 fev. 2021. 